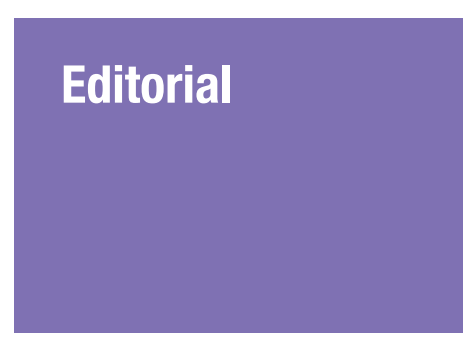

Submitted: 16 Apr 2016 Accepted: 3 May 2017 Online: 18 Aug 2017

\title{
Placebo Controlled Trials: Interests of Subjects versus Interests of Drug Regulators
}

\author{
Teguh Haryo Sasongko ${ }^{1,2}$, Nor Hayati OthmaN ${ }^{1,3}$, Nik Hazlina Nik \\ Hussain ${ }^{1,4}$, Yeong Yeh LeE ${ }^{1,5}$, Sarimah Abdullah ${ }^{1,6}$, Azlan Husin ${ }^{1,5}$, \\ Hans VAN Rostenberghe ${ }^{1,7}$
}

1 Human Research Ethics Committee, Universiti Sains Malaysia, Department of Paediatrics, School of Medical Sciences, Universiti Sains Malaysia, 16150 Kubang Kerian, Kelantan, Malaysia

2 Human Biology Division, School of Medicine, International Medical University, Bukit Jalil, 57000 Kuala Lumpur, Malaysia

3 Department of Pathology, School of Medical Sciences, Universiti Sains Malaysia, 16150 Kubang Kerian, Kelantan, Malaysia

4 Women Health Development Unit, School of Medical Sciences, Universiti Sains Malaysia, 16150 Kubang Kerian, Kelantan, Malaysia

5 Department of Medicine, School of Medical Sciences, Universiti Sains Malaysia, 16150 Kubang Kerian, Kelantan, Malaysia

6 Biostatistics Unit, School of Medical Sciences, Universiti Sains Malaysia, 16150 Kubang Kerian, Kelantan, Malaysia

7 Department of Paediatrics, School of Medical Sciences, Universiti Sains Malaysia, 16150 Kubang Kerian, Kelantan, Malaysia

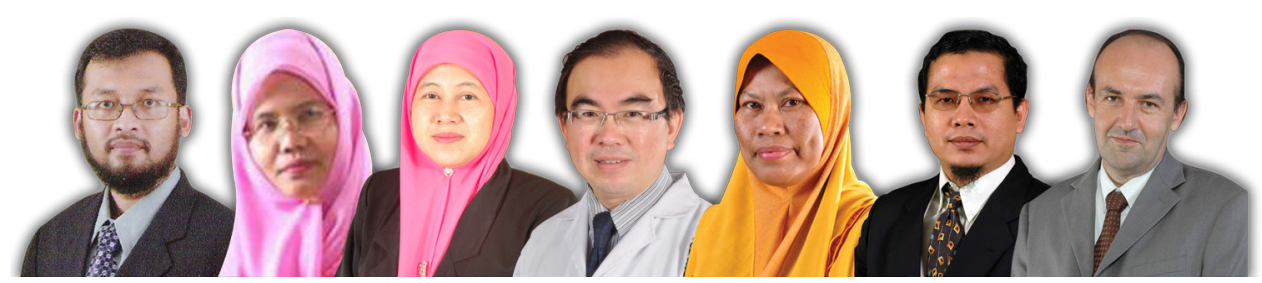

To cite this article: Sasongko TH, Othman NH, Nik Hussain NH, Lee YY, Abdullah S, Husin A, Van Rostenberghe H. Placebo controlled trials: interests of subjects versus interests of drug regulators. Malays $J$ Med Sci. 2017;24(4):1-4. https://doi.org/10.21315/mjms2017.24.4.1

To link to this article: https://doi.org/10.21315/mjms2017.24.4.1

\section{Abstract}

The use of placebo-controlled trials in situations where established therapies are available is considered ethically problematic since the patients randomised to the placebo group are deprived of the beneficial treatment. The pharmaceutical industry and drug regulators seem to argue that placebo-controlled trials with extensive precautions and control measures in place should still be allowed since they provide necessary scientific evidence for the efficacy and safety of new drugs. On the other hand, the scientific value and usefulness for clinical decision-making may be much higher if the new drug is compared directly to existing therapies. As such, it may still be unethical to impose the burden and risk of placebo-controlled trials on patients even if extensive precautions are taken. A few exceptions do exist. The use of placebo-controlled trials in situations where an established, effective and safe therapy exists remains largely controversial.

Keywords: randomised controlled trial, placebo, research ethics, ethics, institutional review board, ethics committee 


\section{Background}

A controversy exists about the ethics of placebo-controlled trials. Whenever a proven intervention exists, the Declaration of Helsinki 2013 (1), article 33, explicitly discourages the involvement of a placebo in human research but allows an exception in rare occasions when compelling reasons for the use of a placebo exist. Clinicians and Institutional Review Boards (IRBs) may find such compelling reasons to be extremely rare or non-existent. Drug regulators and industries, on the other hand, have repeatedly supported and stated compelling reasons to conduct placebo-controlled trials even in the presence of proven efficacious therapies.

This article aims to explore each of the above viewpoints and correlate them to recent literature on the topic.

\section{Perspective of the Clinician}

A new drug needs to be shown to be superior to, or at least non-inferior to, existing proven effective therapies before a clinician will start to use it. In this regard, placebo-controlled trials do not address the question of superiority or non-inferiority and would therefore offer limited clinical value to clinicians. In addition to limited merit, the danger of administering a placebo for a condition with available efficacious therapies is high. As such, placebo-controlled trials have an unfavourable risk/benefit ratio.

Exceptions include the use of add-on therapies where either the new therapy or the placebo is added to the existing standard of care and situations where an established way of treating patients has been around for a long time but has never been founded on rigorous highquality research. Examples of such exceptions also include medications prescribed off-label.

\section{Perspective from Drug Regulators and Industry}

Drug regulators, such as the European Medicines Agency (EMA), have generated multiple explicit statements defending the use of placebo-controlled trials, even in the presence of proven efficacious treatments $(2,3)$. These statements have been used by the sponsors of clinical trials in an attempt to convince IRBs of the acceptability of certain trials.

It seems that well-respected drug regulators support the industry in their call that placebocontrolled trials are necessary, even if other effective therapies have been established. A critical question arising from this scenario is whether the need for new products, products that are better than the placebo but not proven to be better than existing products, reflects the need of the all-powerful big pharmaceutical industry or the need for public health.

\section{Placebo- versus Active-controlled Trials}

Freedman (4) was among the authors who rejected the use of a placebo for the sole purpose of scientific curiosity or the desire to achieve a clean biological analysis of a specific drug effect.

A lack of assay sensitivity in activecontrolled trials (ACTs) as compared to placebocontrolled trials (PCTs) has been mentioned in ICH-E10, section 1.5 (5). Several authors $(6,7,8)$ have rebutted the assay sensitivity argument in a very convincing way.

Another argument brought up in favour of PCTs was that ACTs do not measure the absolute effect size (ICH-E10 section 2.1.6.2) (5). Howick (7) convincingly argued that this operates on the false assumption of 'additivity'.

Howick (7) also argued against the notion that ACTs are less ethical because they usually involve a larger sample size (ICH-E1o section 2.4.7.2) (5). If a PCT is designed to detect a difference that is the same size as the equivalence margin, it will require a sample size that is equally as large as an ACT. Moreover, further studies requiring more samples are needed to determine how the new treatment compares with the best existing treatment.

For the industry, it is easier to demonstrate the superiority of a new drug over a placebo than it is to demonstrate the superiority of a new drug over an existing treatment. Of course, the question remains, if it is not superior to an existing drug, then why do we need the new drug? Indeed, it may be increasingly difficult to produce new drugs that are superior to the existing ones; however, from a patient/society point of view, one could argue as to why new drugs are needed that are not superior to the existing ones.

Drug regulators $(2,3)$ may be more inclined to approve or disapprove new drugs based on the results of placebo-controlled trials than on trials comparing two active substances (new and standard). The pharmaceutical industry is an extremely strong driving force for local economies and one might question the amount of pressure this industry can or does impose on legal drug regulators who are directly responsible to the governments of their respective countries. 


\section{Placebo-controlled Trials and the Difference Position}

In most countries, medical doctors are bound by the Declaration of Geneva (9), which states that 'the health of my patient will be my first consideration,' and/or the International Code of Medical Ethics.

Miller and Brody (10) argued that the ethics of research, which governs the researchersubject relationship, are fundamentally different from the ethics of therapy, governing the physician-patient relationship. This is also known as the 'difference position'. In practice, even if we adopt the 'difference position', it is easy to recognise that, in clinical trials, the physician and the researcher are the same person and the patient and the subject are also the same person. Therefore, the ethical norms will be competing at best, where the immediate interests of the therapy and the patients ought to prevail.

Physician-researchers have a fiduciary duty to the patient-subjects, including a duty of care. Extending this argument to the application of placebo-controlled trials, the need for competent care would support restrictions on the use of placebo controls in clinical research. Accordingly, clinical equipoise requires the adoption of an active control (comparator intervention) in clinical trials investigating new treatments for serious conditions for which a proven treatment exists (11). This is also known as the 'similarity position,' which recognises the unified ethics between that of research and that of therapy.

\section{Summary}

The use of placebo-controlled trials when an effective and safe therapy exists remains largely controversial. We argued in this article that PCTs, compared to ACTs, might have an unacceptable risk/benefit ratio for clinicians and patients, although PCTs may be useful for industry and drug regulators.

\section{Competing Interests}

All authors are medical members of the IRB or human research ethics committee of the Universiti Sains Malaysia.

\section{Acknowledgements}

All authors acknowledge the members of USM Human Research Ethics Committee for their constructive discussions regarding this topic and wish to thank an anonymous reviewer who has generously provided beneficial suggestions to improve the manuscript.

\section{Authors' Contributions}

Conception and design: HVR

Drafting of the article: THS, HVR

Critical revision of the article for important intellectual content: THS, HVR

Final approval of the article: THS, NHO, NHNH, LYY, SA, AH, HVR

\section{Correspondence}

Dr Hans Van Rostenberghe

MD (Ghent, Belgium), Specialist in Pediatrics (Ghent, Belgium)

Chairman,

Human Research Ethics Committee, Universiti Sains

Malaysia

Department of Paediatrics, School of Medical Sciences, Universiti Sains Malaysia

16150 Kubang Kerian, Kelantan, Malaysia.

Tel: +6o 179017848

E-mail: hansvro@usm.my

Alternative e-mail: hansvro@yahoo.co.uk

\section{References}

1. World Medical Association (WMA). 1964-2013. Declaration of Helsinki. [Internet] [cited 12 April 2016]. Available from https://www.wma. net/policies-post/wma-declaration-of-helsinkiethical-principles-for-medical-research-involvinghuman-subjects/

2. The European Agency for the Evaluation of Medicinal Products (EMA). EMEA/CPMP position statement of the use of Placebo in clinical trials with regard to the revised Declaration of Helsinki. 2001. [Internet] [cited 12 April 2016]. Available from http://www.ema.europa. eu/docs/en_GB/document_library/Position_ statement/2009/12/WC500017646.pdf 
3. The European Agency for the Evaluation of Medicinal Products (EMA). Guideline on clinical investigation of medicinal products, including depot preparations in the treatment of schizophrenia. 2012. [Internet] [cited 12 April 2016]. Available from http://www.ema.europa. eu/docs/en_GB/document_library/Scientific_ guideline/2012/10/WC500133437.pdf

4. Freedman B. Placebo-controlled trials and the logic of clinical purpose. IRB. 1990 NovDec;12(6):1-6. https://doi.org/10.2307/3564541

5. The ICH Harmonised Tripartite Guidelines on Choice of Control Group and Related Issues in Clinical Trials (ICH-E10). 20 July 2000. [Internet] [cited 12 April 2016]. Available from http://www.ich.org/fileadmin/Public_Web_Site/ ICH_Products/Guidelines/Efficacy/E10/Step4/ E10_Guideline.pdf

6. Anderson JA. The ethics and science of placebocontrolled trials: assay sensitivity and the DuhemQuine thesis. J Med Philos. 2006 Feb;31(1):6581.https://doi.org/10.1080/03605310500499203
7. Howick J. Questioning the methodologic superiority of 'placebo' over 'active' controlled trials. Am J Bioeth. 2009 Sep;9(9):34-48. https://doi.org/10.1080/15265160903090041

8. Hey SP, Weijer C. Assay sensitivity and the epistemic contexts of clinical trials. Perspect Biol Med. 2013;56(1):1-17. https://doi.org/10.1353/ pbm.2013.0002

9. World Medical Association (WMA). 1948-2006. Declaration of Geneva [Internet] [cited 12 April 2016]. Available from https://www.wma.net/ policies-post/wma-declaration-of-geneva/

10. Miller FG, Brody H. A critique of clinical equipoise: therapeutic misconception in the ethics of clinical trials. The hastings center report. 2003;33(3):19-28. https://doi. org/10.2307/3528434

11. Miller PB, Weijer C. Fiduciary obligation in clinical research. Journal of Law, Medicine \& Ethics. 2006;34(2):424-440. https://doi.org/ 10.1111/j.1748-720X.2006.00049.x 